\title{
ON LATTICE SUMMING OPERATORS
}

\author{
JERZY SZULGA
}

\begin{abstract}
Banach space $E$ and a Banach lattice $L$, necessary and sufficient conditions on $E$ and $L$ are given such that every lattice summing operator $T: E \rightarrow L$, (cf. Introduction) is absolutely summing.
\end{abstract}

1. Introduction. The concept of absolutely summing operators has a certain natural analogue when the range space is a Banach lattice. Namely, an operator $T: E \rightarrow L$ is called lattice summing, if for every sequence $\left(x_{n}\right)$ in $E$ such that $\sum x_{n}$ converges unconditionally, the series $\Sigma\left|T x_{n}\right|$ converges in $L$. Of course, if e.g. $L$ is an $L_{1}$-space or $E$ is an $L_{1}$-space and $L$ is a Hilbert space, then both notions coincide.

The aim of this paper is to characterize all pairs $E, L$ for which this happens.

In 1979 Yanovskii [10] investigated problems related to lattice summability. In particular, he formulated a conjecture that, if all lattice summing operators acting on the same Banach lattice $L$ are absolutely summing, then $L$ is isomorphic to $L_{1}$. However, it follows from our characterization that spaces of cotype 2 have this property. Another by-product of this paper is a still different formulation of the Dubinsky-Pekczyński-Rosenthal property $\Pi_{2}\left(\varrho_{\infty}, E\right)=B\left(\varrho_{\infty}, E\right)$.

2. Notations and preliminary facts. Throughout this paper $E, F$ and $L, K$ denote infinite-dimensional Banach spaces and Banach lattices, respectively. $E^{\prime}$ is the norm dual of $E$ and $p^{\prime}$ stands for the exponent dual to $p \in[1, \infty]$. The natural basis in $l_{p}$, $1 \leqslant p<\infty$, is denoted by $e_{1}, e_{2}, \ldots$. For a finite sequence $\left(x_{i}\right)$ in $E$ we set

$$
w_{p}\left(\left\{x_{i}\right\}\right)=\sup \left\{\left(\sum\left|\left\langle x^{\prime}, x_{i}\right\rangle\right|^{p}\right)^{1 / p}: x^{\prime} \in E^{\prime},\left\|x^{\prime}\right\| \leqslant 1\right\} .
$$

An operator $T: E \rightarrow F$ is called $p$-absolutely summing if there exists a constant $C>0$ such that, for all $x_{1}, \ldots, x_{n} \in E$,

$$
\left(\sum\left\|T x_{i}\right\|^{p}\right)^{1 / p} \leqslant C w_{p}\left(\left\{x_{i}\right\}\right) .
$$

The smallest $C$ is denoted by $\Pi_{p}(T)$ and is the norm in the Banach space $\Pi_{p}(E, F)$ of all $p$-absolutely summing operators.

An operator $T: E \rightarrow L$ is called lattice summing if, for some $C>0,\left\|\Sigma\left|T x_{i}\right|\right\| \leqslant$ $C w_{1}\left(\left\{x_{i}\right\}\right)$ for all $x_{1}, \ldots, x_{n} \in E$. The smallest $C$, denoted by $\Lambda(T)$, is the norm in the Banach space $\Lambda(E, L)$ of all lattice summing operators from $E$ into $L$. Clearly $\Lambda(T) \leqslant \Pi_{1}(T)$.

Received by the editors October 19, 1981 and, in revised form, April 23, 1982 and June 22, 1982. 1980 Mathematics Subject Classification. Primary 47B10; Secondary 46B20.

Key words and phrases. p-absolutely summing operators, majorizing operators, Banach lattices, cotype of Banach spaces. 
The following easy construction shows how lattice summing operators lead to absolutely summing operators. Let $\left(L, y^{\prime}\right)$, where $y^{\prime} \in L^{\prime}, y \geqslant 0$, denotes the completion of $L$ with respect to the seminorm $\left\langle y^{\prime},|\circ|\right\rangle .\left(L, y^{\prime}\right)$ is isomorphic to $L_{1}$ and the natural lattice homomorphism $I_{y^{\prime}}: L \rightarrow\left(L, y^{\prime}\right)$ is continuous.

Proposition 2.1. The following assertions are equivalent for an operator $T: E \rightarrow L$ :

(i) $T$ is lattice summing;

(ii) for each $y^{\prime} \geq 0, I_{y^{\prime}} \circ T$ is absolutely summing;

(iii) for every, or equivalently, for just one infinite-dimensional $L_{1}$-space and for each positive operator $S: L \rightarrow L_{1}, S \circ T$ is absolutely summing.

We omit a standard proof. In order to give examples of lattice summing operators we state the following result. First we recall that an operator $T: E \rightarrow L$ is said to be majorizing if

$$
M(T):=\sup \left\{\left\|\sup _{i}\left|T x_{i}\right|\right\|:\left\|x_{i}\right\| \leqslant 1, i=1, \ldots, n\right\}
$$

is finite. We denote by $M(E, L)$ the Banach space of such operators.

PROPOSITION 2.2. Let $T: E \rightarrow L$. T is lattice summing if and only if for each operator $U: l_{\infty} \rightarrow E, T \circ U$ is majorizing.

Proof. We have

$$
\begin{aligned}
\Lambda(T) & =\sup _{n} \sup \left\{\left\|\sum_{i=1}^{n}\left|T x_{i}\right|\right\|: w_{1}\left(\left\{x_{i}\right\}\right) \leqslant 1,\left\{x_{i}\right\} \subset E\right\} \\
& =\sup _{n} \sup \left\{\left\|\sup \left\{\left|T a_{i} x_{i}\right|:\left|a_{i}\right| \leqslant 1\right\}\right\|: w_{1}\left(\left\{x_{i}\right\}\right) \leqslant 1\right\} \\
& =\sup _{n} \sup \left\{\left\|\sup \left\{|T S u|: u \in l_{\infty}^{n},\|u\|_{\infty} \leqslant 1\right\}\right\|: S: l_{\infty}^{n} \rightarrow E,\|S\| \leqslant 1\right\} \\
& =\sup \left\{M(T S): S: l_{\infty} \rightarrow E,\|S\| \leqslant 1\right\} . \quad \text { Q.E.D. }
\end{aligned}
$$

COROLlaRY 2.1. Each majorizing operator $T: E \rightarrow L$ is lattice summing and $\Lambda(T)$ $\leqslant M(T)$. If $E=l_{\infty}$ we have more: $M\left(l_{\infty}, L\right)=\Lambda\left(l_{\infty}, L\right)$.

Let us recall a few notions which play important roles in what follows.

By definition, $E$ is of cotype $q$, where $q \geqslant 2$, if there exists a $C>0$ such that for all finite sets $\left(x_{i}\right) \subseteq E$

$$
\left(\sum\left\|x_{i}\right\|^{q}\right)^{1 / q} \leqslant C \int_{0}^{1}\left\|\sum r_{i}(t) x_{i}\right\| d t
$$

where $r_{1}, r_{2}, \ldots$ are Rademacher functions. We say that $L$ is $p$-concave, where $p \in[1, \infty)$, if for a $C>0$,

$$
\left(\sum\left\|x_{i}\right\|^{p}\right)^{1 / p} \leqslant C\left\|\left(\sum\left|x_{i}\right|^{p}\right)^{1 / p}\right\|
$$

for all finite choices of $\left\{x_{i}\right\} \subseteq E$. Here

$$
\left(\sum\left|x_{i}\right|^{p}\right)^{1 / p}=\sup \left\{\sum a_{i} x_{i}: a_{i} \in \mathbf{R}, \sum\left|a_{i}\right|^{p^{\prime}} \leqslant 1\right\}
$$


(cf. [3 or 5] for details). Note if $L$ is 1-concave, then it is isomorphic to an $L_{1}$-space (cf. e.g. [9]). We say that $F$ is finitely representable in $E$ (respectively, $K$ is lattice finitely representable in $L$ ) if there exists $\delta>0$ such that, for each finite-dimensional subspace $F_{0} \subseteq F$ (respectively, sublattice $K_{0} \subseteq K$ ), one can find an isomorphism $I_{0}: F_{0} \rightarrow F\left(\right.$ respectively $I_{0}: K_{0} \rightarrow L$ ) satisfying

$$
\delta^{-1}\|x\| \leqslant\left\|I_{0} x\right\| \leqslant \delta\|x\|
$$

for all $x \in F_{0}$ (respectively, $x \in K_{0}$ ).

\section{Main result.}

THEOREM 3.1. The following conditions are equivalent:

(I) $\Lambda(E, L)=\Pi_{1}(E, L)$;

(II) $M(E, L) \subseteq \Pi_{1}(E, L)$;

(III) there exists $p \in[1,2]$ such that both $\Pi_{p^{\prime}}\left(\mathscr{L}_{\infty}, E\right)=B\left(\mathscr{L}_{\infty}, E\right)$ and $L$ is p-concave.

REMARK. We identify $\infty$-absolutely summing and bounded operators. The following is an adaptation of what we need from Rosenthal [8] and Maurey and Pisier [7].

Proposition 3.1. Let $p \in(1,2]$. The following properties of a Banach space $E$ are equivalent:

(i) $M\left(E, l_{p}\right) \subseteq \Pi_{1}\left(E, l_{p}\right)$;

(ii) there exists a $C>0$ such that

$$
\sum_{j}\left(\sum_{i}\left|\left\langle x_{i}^{\prime}, x_{j}\right\rangle\right|^{p}\right)^{1 / p} \leqslant C w_{1}\left(\left\langle x_{j}\right\rangle\right)\left(\sum\left\|x_{i}^{\prime}\right\|^{p}\right)^{1 / p}
$$

for all $x_{1}, \ldots, x_{n} \in E$ and $x_{1}^{\prime}, \ldots, x_{m}^{\prime} \in E^{\prime}$;

(iii) for every Banach space $F, \Pi_{1}(E, F)=\Pi_{p}(E, F)$;

(iv) $\Pi_{p^{\prime}}\left(\complement_{\infty}, E\right)=B\left(\complement_{\infty}, E\right)$.

Moreover, if $p<2$, then each of the above conditions is equivalent to

(v) $p^{\prime}>q_{E}:=\inf \left\{q: \Pi_{q}\left(\complement_{\infty}, E\right)=B\left(\complement_{\infty}, E\right)\right\}$.

The implications (ii) $\Leftrightarrow$ (iii) $\Leftrightarrow$ (iv) are in Proposition 3 in [8]. The fact that infimum $q_{E}$ is never attained for $q>2$, which is precisely what (v) says, is due to Maurey and Pisier [7]. Finally, (ii) is just a reformulation of (i) expressed in terms of finite rank operators.

Lemma 3.1. Let $M(E, L) \subseteq \Pi_{1}(E, L)$. If $F$ is finitely representable in $E$ and $K$ is lattice finitely representable in $L$ then $\Pi_{1}(T) \leqslant C M(T)$ for all compact operators $T: F \rightarrow K$, where the constant $C$ does not depend on the operators $T$.

Proof. Let $M^{c}$ and $\Pi_{1}^{c}$ denote the classes of compact operators in $M$ and $\Pi_{1}$, respectively. A standard argument shows that $M(E, L) \subseteq \Pi_{1}(E, L)$ implies that $M^{c}(E, K) \subseteq \Pi_{1}^{c}(E, K)$. In particular, by the "local reflexivity principle" (cf. [4, Proposition I. 4]), $M^{c}\left(E, K^{\prime \prime}\right) \subseteq \Pi_{1}^{c}\left(E, K^{\prime \prime}\right)$. Finally, repeating the usual procedure, however, making use of the extension property of majorizing operators (as it is stated in [9, Proposition IV. 3.10]), we infer that $M^{c}(F, K) \subseteq \Pi_{1}^{c}(F, K)$. Q.E.D. 
COROLlaRy 3.1. If $l_{q}$ is finitely representable in $E$, where $1<q \leqslant \infty$, and $M(E, L)$ $\subseteq \Pi_{1}(E, L)$, then $L$ is $q^{\prime}$-concave. In particular, by Dvoretzky's theorem [2], $M(E, L) \subseteq \Pi_{1}(E, L)$ shows $L$ to be 2-concave.

Proof. In fact, by Lemma 3.1 we may, and do, assume that $M\left(l_{q}, L\right) \subseteq \Pi_{1}\left(l_{q}, L\right)$; hence for some $C>0, \Pi_{1}(T) \leqslant C M(T)$, for each operator of the form $T=$ $\sum_{l=1}^{n} e_{1} \otimes y_{i}: l_{q} \rightarrow L$. But

$$
\begin{aligned}
& \Pi_{1}(T) \geqslant \sup \left\{\sum\left|a_{i}\right|\left\|y_{i}\right\|: w_{1}\left(\left\{a_{i} e_{i}\right\}\right) \leqslant 1\right\}=\left(\sum\left\|y_{i}\right\|^{q^{\prime}}\right)^{1 / q^{\prime}} \\
& M(T) \leqslant\left\|\sup \left\{\left|\sum\left\langle e_{i}, x\right\rangle y_{i}\right|:\|x\| \leqslant 1\right\}\right\|=\left\|\left(\sum\left|y_{i}\right|^{q^{\prime}}\right)^{1 / q^{\prime}}\right\| .
\end{aligned}
$$

Combining these estimates we get the desired condition. Q.E.D.

Proof of TheOREM 3.1. (I) $\Rightarrow$ (II) is an immediate consequence of Proposition 2.2 .

(II) $\Rightarrow$ (III). Let $p_{L}:=\inf \{p: L$ is $p$-concave $\}$.

By [4. Theorem II. 2], $l_{p_{l}}$ is lattice finitely representable in $L$. Hence, by Lemma 3.1 and Proposition 3.1, $\Pi_{p_{t}}\left(\varrho_{\infty}, E\right)=B\left(£_{\infty}, E\right)$.

Now we examine three cases: $p_{L}=2, p_{L} \in(1,2)$ and $p_{L}=1$. The first yields (III) with $p=2$, in view of Corollary 3.1. In the second case we apply condition (v) of Proposition 3.1 so that $p_{L}^{\prime}>q_{E}$ and this implies (III) with $p \in(1,2) . p_{L}=1$ carries no information about $E$, however, if $q_{E}<\infty$, then again $q_{E}^{\prime}>p_{L}=1$ and we proceed as before in order to obtain (III) for a $p \in(1,2]$. On the other hand, if $q_{E}=\infty$ or, equivalently, if $l_{\infty}$ is finitely representable in $E$ (see [7]), then by Corollary $3.1, L$ is 1 -concave (note that $p_{L}=1$ does not imply 1 -concavity, in general). This completes the proof of (II) $\Rightarrow$ (III).

$(\mathrm{III}) \Rightarrow(\mathrm{I})$. Let $p \in[1,2]$ be such that $\Pi_{p^{\prime}}\left(\mathscr{L}_{\infty}, E\right)=B\left(\mathscr{E}_{\infty}, E\right)$ and let $L$ be $p$-concave. Hence, by Proposition $3.1, \Pi_{1}(E, L)=\Pi_{p}(E, L)$. Therefore, by (III), there exist constants $C$ and $C_{1}$ such that for each $T: E \rightarrow L$ we have

$$
\begin{aligned}
\Pi_{1}(T) & \leqslant C \Pi_{p}(T) \leqslant C C_{1} \sup \left\{\left\|\left(\sum\left|T x_{i}\right|^{p}\right)^{1 / p}\right\|: w_{p}\left(\left\{x_{i}\right\}\right) \leqslant 1\right\} \\
& \leqslant C C_{1} \sup \left\{\left\|\int_{0}^{1}\left|\sum T x_{i} f_{i}(t)\right| d t\right\|: w_{1}\left(\sum x_{i} f_{i}\right) \leqslant 1\right\}
\end{aligned}
$$

where $\left(f_{i}\right)$ is a sequence of independent standard real $p$-stable random variables on $[0,1]$ and

$$
w_{1}(f)=\sup \left\{\int_{0}^{1}\left|\left\langle x^{\prime}, f(t)\right\rangle\right| d t: x^{\prime} \in E^{\prime}, .\left\|x^{\prime}\right\| \leqslant 1\right\}
$$

for $E$-valued function $f$ (cf. [3]). By routine measure theory one can check that

$$
\Lambda(T)=\sup \left\|\int_{0}^{1}|T f(t)| d t\right\|
$$

where the supremum is taken over all finite rank measurable functions with $w_{1}(f) \leqslant 1$. Hence, $\Pi_{1}(T) \leqslant C C_{1} \Lambda(T)$ for all operators $T: E \rightarrow L$. This completes the proof of (III) $\Rightarrow(I)$. Q.E.D. 
4. Comments and corollaries. By Maurey-Pisier [7], $q_{E}=\inf \{q: E$ is of cotype $q\}$. Hence, in virtue of Proposition 3.1, the third assertion of Theorem 3.1 can be rephrased in the following manner:

(III') One of the following conditions is satisfied:

(i) $l_{\infty}$ is finitely representable in $E$ and $L$ is isomorphic to $L_{1}$.

(ii) there exist $p, q, 1 \leqslant p<q^{\prime} \leqslant 2$, such that $E$ is of cotype $q$ and $L$ is $p$-concave,

(iii) $\Pi_{2}\left(E_{\infty}, E\right)=B\left(\varrho_{\infty}, E\right)$ and $L$ is 2 -concave.

If $E$ is a Banach lattice, then the above takes a much simpler appearance (cf. [6]):

(III) There exist $p, q$ such that either $p=q^{\prime}=1$ or $p=q^{\prime}=2$ or $1 \leqslant p<q^{\prime} \leqslant 2$, and $E$ is of cotype $q$ and $L$ is $p$-concave.

Corollary 4.1. $\Lambda(L, L)=\Pi_{1}(L, L)$ if and only if $L$ is 2-concave.

Corollary 4.2. For a Banach space $E, \Pi_{2}\left(\varrho_{\infty}, E\right)=B\left(\varrho_{\infty}, E\right)$ if and only if for some constant $C>0$ for each operator $U: E^{\prime} \rightarrow l_{1}$,

$$
\int_{0}^{1}\left\|\sum U x_{i}^{\prime} r_{i}(t)\right\| d t \leqslant C\|U\| \sup \left\{\left(\sum\left\|V x_{i}^{\prime}\right\|^{2}\right)^{1 / 2}: V: E^{\prime} \rightarrow l_{1},\|V\| \leqslant 1\right\}
$$

for all $x_{1}^{\prime}, \ldots, x_{n}^{\prime} \in E^{\prime}$.

\section{REFERENCES}

I. E. Dubinsky, A. Pelczyński and H. P. Rosenthal, On Banach spaces for which $\mathrm{II}_{2}\left(E_{\infty}, E\right)=$ $B\left(E_{\propto}, E\right)$. Studia Math. 44 (1972), 617-634.

2. A. Dvoretzky. Some results on convex hodies in Banach spaces (Proc. Sympos. Linear Spaces. Jerusalem, 1961), Jerusalem Academic Press, Jerusalem; Pergamon, Oxford, 1961, pp. 123-160.

3. J. L. Krivine, Théorèmes de factorization dans les espaces réticules, Séminaire Maurey-Schwartz, 1973-74. exp. 22-23.

4. Sous espaces de dimension finie des espaces de Banach réticules, Ann. of Math. (2) 104 (1976), 1-29.

5. J. Lindenstraus and L. Tzafriri, Classical Banach spaces, Vol. II, Springer-Verlag, Berlin, Heidelberg and New York. 1979

6. B. Maurey, Type et cotype dans les espaces munis de structures locales inconditionneles, Sem. Maurey-Schwartz, 1973-74, exp. 24-25.

7. B. Maurey and G. Pisier. Séries de variables aleatoires vectorielles independantes et propriètes géométriques des espaces de Banach. Studia Math. 58 (1976), 45-90.

8. H. P. Rosenthal, On subspaces of $L^{p}$. Ann of Math. (2) 97 (1973). 344-373.

9. H. H. Schaeffer, Banach lattices and positive operators, Springer-Verlag. Berlin, Heidelberg and New York, 1974.

10. L. P. Yanovskii, On summing and lattice summing operators and characterization of AL-spaces, Sibirsk. Mat. Ž. 20 (1979), 401-408. (Russian)

Institute of Mathematics, Wrockaw University, Pl. Grunwaldzki 2/4,50384 Wrockaw, POLAND 